\title{
Equipamento portátil para ensaios simultâneos de teste de vigilância psicomotora e pupilometria
}

John Kennedy Schettino de Souza, Giuliano Emerenciano Ginani, Sabine Pompéia, Jerome Baron, Carlos Julio Tierra-Criollo*

Resumo Neste artigo é descrito um sistema com potencial para identificar a privação do sono, que, com base no levantamento bibliográfico realizado, ainda não foi abordado na literatura. Este sistema integra simultaneamente duas metodologias, o teste de vigilância psicomotora (Psychomotor vigilance test, $\mathrm{PVT}$ ) e a pupilometria, que se destacam no estudo da privação do sono. Entretanto, para atender às peculiaridades destas metodologias, permitindo que coexistam em um único sistema, algumas adaptações foram realizadas em seus procedimentos. Esta integração poderá garantir não só a complementariedade de indicadores que torna a identificação da privação do sono mais robusta, assim como estabelecer a equalização do estado psicofisiológico do sujeito, o que não é possível em testes realizados com defasagem temporal. Neste estudo, a validação das métricas do sistema foi realizada com sujeitos em estado de alerta. Os resultados mostraram-se coerentes com a literatura. Entretanto, algumas métricas apresentam um deslocamento em seus valores médios, que segundo as avaliações realizadas são determinadas pelas exigências técnicas do sistema. Os resultados obtidos nesta avaliação, somados à crescente demanda de ferramentas de aplicação em larga escala e que possam ser utilizadas além dos limites laboratoriais para estudos em distúrbios e privação do sono, apontam este sistema como uma potencial ferramenta. Entretanto, será necessário o estabelecimento de um experimento rigoroso, para avaliar se os indicadores oriundos das métricas do sistema permitem a identificação robusta da privação do sono.

Palavras-chave Pupila, Pupilometria, Pupilômetro, Tempo de reação, PVT.

\section{Portable equipment for simultaneous assays of psychomotor vigilance test and pupillometry}

\begin{abstract}
This paper describes a system with potential for identification of sleep deprivation, which, based on our bibliographical survey, has not yet been described in the literature. The system combines two methodologies, i. e., Psychomotor vigilance test (PVT) and pupillometry, which are among the leading methods for the study of sleep deprivation. However, due to peculiarities of both methodologies, some adaptations were made in their procedures to allow them to co-exist in the same system. Such integration may not only ensure the complementarity of indexes, making the identification of sleep deprivation more solid, but also set up the equalization of the subject's psycho-physiological state, which is not possible in tests performed with a time lag. In this study, the performance of measurements provided by the system was assessed in subjects on alert. However, some measurements present a displacement with respect to their average values, which, according to assessment, are determined by system's technical requirements. The results obtained in this assessment, combined with the increasing demand for large scale application tools, able to be used outside the limits of the laboratory environment for studies in sleep deprivation disorders, point to this system as a potential tool. However, the undertaking of a rigorous experiment is necessary to assess whether the indexes obtained by the system allow the robust identification of sleep deprivation.
\end{abstract}

Keywords Pupil, Pupillometry, Pupillometer, Reaction time, PVT. 


\section{Introdução}

Pesquisas realizadas pelo Center for Narcolepsy, Sleep and Health Research, University of Illinois at Chicago Colleges of Nursing and Medicine estimam que entre 40 a 70 milhões de americanos apresentam problemas de ordem crônica ou de intermitência do sono (Center..., 2012). Estes problemas se manifestam independentemente do sexo, raça ou grupo sócio econômico sendo agravados com a idade. $\mathrm{O}$ desconhecimento, por parte do sujeito, do distúrbio do sono e a consequente falta de tratamento tem um forte impacto social refletindo no aumento da morbidade (Liang et al., 2012) e mortalidade, na baixa qualidade de vida (Hoge et al., 2011), na menor produtividade (Porcu et al., 1998) e no comprometimento da segurança pública (Wilhelm et al., 2009) devido à alta incidência de acidentes relacionados com a sonolência excessiva (Smolensky et al., 2010).

Diversos são os métodos aplicados no estudo de distúrbios do sono, sendo o Multiple sleep latency test (MSLT) a referência (padrão ouro) na quantificação objetiva da sonolência e no diagnóstico de narcolepsia (Bitsios et al., 2006; Liu et al., 2008), fundamentando-se em indicadores de natureza fisiológica (respiração, cardio-vascular, oculomotora, atividade elétrica cerebral, condução da pele e temperatura). Entretanto, o MSLT tem no seu custo o fator impeditivo para a sua aplicação em larga escala, demandando um quadro de profissionais especializados e um grande número de equipamentos: eletroencefalógrafo (EEG), eletrocardiógrafo (ECG), eletrooculógrafo (EOG), eletromiógrafo (EMG), oxímetro, medidores de fluxo e sons respiratórios (Carskadon et al., 1986).

Existem métodos alternativos onde por meio de formulários, contendo uma série de situações propostas, o sujeito estima o seu grau de sonolência. Embora atrativos pelo seu baixo custo, estes métodos denominados subjetivos, são vulneráveis no seu diagnóstico, pois o sujeito pode superestimar ou subestimar suas impressões dependendo do seu estado psicofisiológico (Curcio et al., 2001). Por outro lado, existem métodos objetivos e de natureza comportamental, tendo como destaque o teste de vigilância psicomotora (Psychomotor vigilance test, PVT) devido a sua alta sensibilidade à privação do sono (Basner e Dinges, 2011). Segundo Basner e Dinges (2011) basta um computador pessoal para realizar a estimulação visual e a captura do tempo de resposta. A grande vantagem da abordagem comportamental é a fácil aplicabilidade dos métodos. Entretanto, tem como principal limitação a dependência do estado motivacional do indivíduo (Curcio et al., 2001). Já entre os métodos objetivos e de natureza fisiológica destaca-se a pupilometria (oscilação da pupila). Entretanto, estes métodos alternativos são indicados apenas como auxiliares (McLaren et al., 2002) ou para avaliação específica (Caffier et al., 2003, 2005) no estudo de problemas associados à sonolência.

Uma estratégia muito utilizada na identificação de privação do sono, que propicia uma complementariedade nos indicadores, é a aplicação de métodos de natureza subjetiva e objetiva ao longo do exame (Franzen et al., 2008). Entretanto, a diferença temporal (não simultâneas) entre as aplicações inviabiliza uma equalização nas condições psicofisiológicas do sujeito. Estudo bem sucedido de tal equalização foi aplicado por Bernhardt et al. (1996) em psicologia social. Nele, o eyetraker comercial ISCAN modelo RK-426, Cambridge, MA foi integrado a um computador pessoal para estimulação visual/auditiva com o monitoramento simultâneo do diâmetro da pupila. Já em 2007 num estudo para determinação do estado de sedação do paciente, Patil et al. (2007) integraram, em um único dispositivo, mecanismos para a estimulação visual, a monitoração da fala e da dinâmica da pupila.

Devido à grande aplicação do PVT e da pupilometria no estudo de privação do sono, a proposta deste trabalho é desenvolver um sistema portátil de fácil operação, que além de garantir a equalização das condições psicofisiológicas do sujeito, obtenha simultaneamente os indicadores objetivos de natureza comportamental (PVT) e fisiológica (pupilometria). Um ensaio experimental, com sujeitos em estado de alerta, foi realizado com objetivo de avaliar (1) a possibilidade de aquisição simultânea de métricas responsáveis pela geração dos indicadores do PVT e da pupilometria e se (2) tais métricas apresentam resultados compatíveis com a literatura.

\section{Materiais e Métodos}

\section{Sujeitos}

A amostra consistiu de 19 indivíduos saudáveis do sexo masculino com média de idade 24,5 variando entre 21 e 33 anos, com um mínimo de 12 anos de escolaridade. Os sujeitos não tinham problemas psiquiátricos, incluindo a dependência de drogas ou problemas clínicos que poderiam interagir com os efeitos da privação do sono, como a epilepsia, distúrbios de aprendizagem, distúrbios do sono (confirmada pela polissonografia), tampouco problemas auditivos ou déficits visuais não corrigidos. Nenhum dos indivíduos era trabalhador de turno ou viajara em vôos transmeridianos no mês anterior ao experimento, para evitar possíveis distúrbios dos ritmos circadianos. Eles 
tinham um índice de massa corporal entre 20 e 25, eram não fumantes e não estavam submetidos a medicação no momento do estudo. Os voluntários não estavam nos extremos da dimensão cronotipo medida pelo questionário de Horne e Ostberg (Benedito-Silva et al., 1990; Horne e Ostberg, 1976) (avalia o padrão do ritmo circadiano da pessoa que pode ser matutino, vespertino ou indiferente), e tinham um padrão de sono normal, isto é, eles dormiam 6-9 horas/noite e, geralmente, adormeciam entre as $23 \mathrm{~h} 00 \mathrm{e} 01 \mathrm{~h} 00$. Todos eles tinham escores dentro da faixa normal no teste de inteligência não-verbal de Matrizes Progressivas de Raven (Raven et al., 1988).

\section{Procedimento}

Este estudo foi aprovado pelo Comitê de Ética da Universidade Federal de São Paulo (UNIFESP; 0763/10) e todos os sujeitos assinaram o termo de consentimento livre e esclarecido. Para a seleção dos voluntários foi realizada uma entrevista estruturada, incluindo a história clínica, escalas de depressão e ansiedade (STAI-Traitscale (Gorenstein et al., 1995; Spielberger et al., 1970) e Beck Depression Inventory (Beck et al., 1961; Gorenstein et al., 1995)), sonolência (Epworth Sleepiness Scale (Johns, 1991)) e triagem para distúrbios do sono e cronotipo (questionário de Horne e Ostberg (Benedito-Silva et al., 1990; Horne e Ostberg, 1976)).

No dia do experimento, os voluntários chegaram ao laboratório por volta das $07 \mathrm{~h} 00$ após uma noite de sono, sendo esta monitorada por meio de um actígrafo (Motionlogger Actigraph/Octogonal Basic, Ambulatory Monitoring, Inc., Ardsley, NY, USA). Segundo Telles et al. (2011), com o actígrafo é possível realizar um levantamento dos períodos de sono e vigília do sujeito para estudo do ciclo sono-vigília e seus ritmos circadianos. Antes do experimento os voluntários ingeriram um desjejum padronizado, sem cafeína.

A temperatura e a luminosidade da sala de testes foram mantidas constantes durante todo o experimento. Um luxímetro digital (Modelo 615, B\&K Precision, USA) foi posicionado próximo do olho exposto do voluntário e foi obtida uma iluminância próxima de 20 lux.

\section{Sistema integrado de estimulação visual e de aquisição de imagens}

O sistema desenvolvido para este experimento é constituído de um computador pessoal (Figura 1a), uma câmera de vídeo FireFly ${ }^{\mathbb{B}}$ MV (Point Grey Research, Richmond, Canada, USA, www.ptgrey.com/products/ fireflymv/) que se comunica via padrão IEEE 1394 e

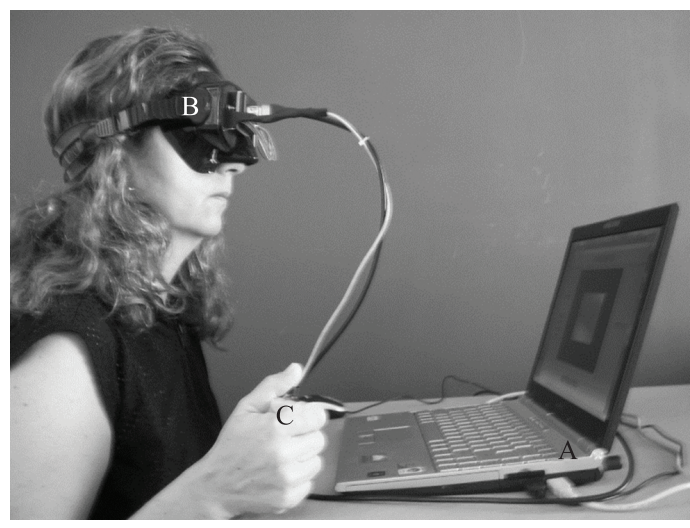

Figura 1. Sistema integrado de estimulação visual e de aquisição de imagens: (a) Microcomputador utilizado para controle do ensaio e recepção e armazenamento de dados; (b) Máscara com câmera de vídeo; (c) Botão de resposta a estimulação. Exemplificação típica de preparação de um sujeito para realização de ensaio: colocação da máscara, posicionamento em frente ao terminal de vídeo e sustentação do botão de resposta ao estímulo visual.

Figure 1. Combined system of visual stimulation and image acquisition: (a) Computer used for the experiment's control as well as for receiving and storing data; (b) Mask with video camera; (c) Stimulation response switch. Typical exemplification of the subject's preparation for the experiment: placement of the mask, positioning in front of the video terminal and e holding of the visual stimulation response switch.

realiza a captura e digitalização da imagem de forma autônoma, dispensando o uso placa de aquisição de imagem no computador. Esta câmera encontra-se montada numa máscara de mergulho (Figura 1b) adaptável à anatomia humana. Para captura do tempo de reação o sistema possui um botão (Figura 1c), que deve ser mantido em uma das mãos do sujeito, segundo sua preferência, e deve ser pressionado na ocorrência da estimulação visual.

A iluminação da cena é realizada por fontes de luz IR (infra red $\lambda=850 \mathrm{~nm}$ ) que minimiza o efeito da modulação da pupila devido à luz visível (Figura 2a). A potência de cada LED (light-emitting diode) IR (Figura 2a-L1-L4) foi fixada em $0,12 \mathrm{~mW} / \mathrm{cm}^{2}$, o que garante a qualidade da iluminação da cena não excedendo aos limites estabelecidos pelo International Commission on Non-Ionizing Radiation Protection $\left(10 \mathrm{~mW} / \mathrm{cm}^{2}\right.$ por um período de 17 minutos) (International..., 1997).

O sistema monocular foi projetado originalmente para monitorar o olho direito do sujeito. No ensaio exclusivo de pupilometria a lente esquerda da máscara foi substituída por um tampão opaco (Figura 2b) para garantir o ambiente escotópico. Entretanto, para viabilizar a integração com o PVT onde o sujeito necessita de um evento visual para disparar a atividade motora, o tampão deve ser retirado (Figura 2c). Para minimizar uma possível modulação da pupila devido ao estímulo visual foi adotada, diferentemente do cronômetro 
utilizado originalmente no VRT (visual reaction time) descrito em Dinges e Powell (1985), uma estimulação IR, que só é percebida pelo sujeito na imagem capturada pelo sistema. Os dois LEDs IR (L1 e L2 Figura 2a), dispostos horizontalmente na placa de circuito impresso, são utilizados inicialmente para iluminação da cena. Entretanto, devido à sensibilidade da câmera ao IR, dois pontos (Figura 3a) são formados na imagem sobre a pupila que correspondem ao reflexo da córnea dos respectivos feixes dos LEDs IR. O início da estimulação ocorre quando a fonte de iluminação passa dos LEDs dispostos horizontalmente (L1 e L2 - Figura 2a) para os LEDs dispostos verticalmente (L3 e L4 - Figura 2a) resultando na imagem da Figura 3b. Esta estimulação é extinta quando o sujeito pressiona o botão.

A iluminação da cena assim como a estimulação visual é comandada pelo software, via canais de Input/Output 1 e 2 (padrão elétrico TTL - transistor-transistor logic) presentes na câmera. Por outro lado, a captura da resposta ao estímulo é realizada por meio do canal Input/Output 0. Todos estes sinais são gravados numa região da imagem

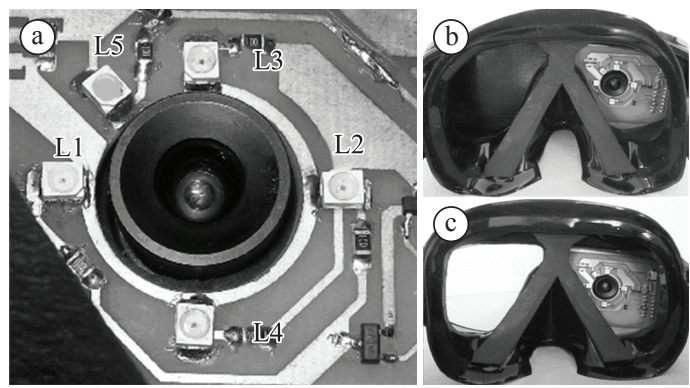

Figura 2. a) Placa de circuito impresso com câmera, LEDs IR (L1, L2, L3 e L4) de iluminação e estimulação (dupla função). O LED branco L5 é utilizado para estimulação visual no espectro visível. b) Manutenção do ambiente escotópico com tampão e placa de circuito impresso com câmera; c) Janela para observação da estimulação visual na tela do computador.

Figure 2. a) Printed circuit board with camera, infrared lighting and stimulation LEDS (L1,L2, L3 and L4) (double function). The white LED (L5) is used for the visual stimulation in the visible spectrum. b) Scotopic environment kept with black rubber sheet and printed circuit board with camera; c) Visual stimulation's observation window on the computer screen.
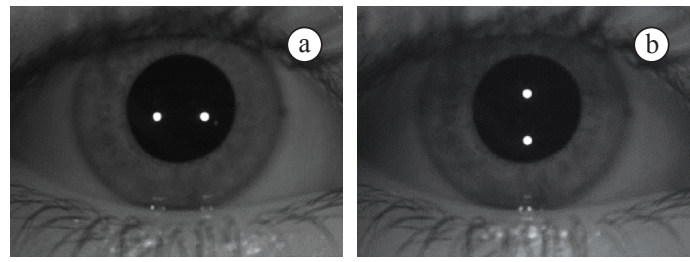

Figura 3. a) Iluminação da cena e respectivo reflexo corneal. b) Iluminação da cena e início da estimulação visual.

Figure 3. a) Scene's lighting and respective corneal reflection. b) Scene's lighting and beginning of the visual stimulation. juntamente com um timestamp que é um marcador de tempo do instante da captura do quadro. O timestamp é fundamental na identificação de falhas de captura, principalmente para sistemas operacionais que não dão suporte a tempo real, que é uma restrição típica do Windows (Microsoft ${ }^{\circledR}$ Corporation, Washington, USA).

O sistema também permite a realização de ensaio de reflexo pupilar à luz (RPL) utilizando o LED L5 da Figura 2a, responsável por uma estimulação visual no espectro visível. Assim como os demais LEDs o L5 é comandado por software via o canal Input/Output 3 da câmera.

O software foi escrito em linguagem gráfica de programação LabView 8.5, desenvolvido pela National Instruments (www.ni.com, Texas, USA) e testado para versões XP e Vista do sistema operacional Windows.

\section{Metodologia para obtenção das métricas do sistema}

A robustez dos indicadores para o diagnóstico da privação do sono é diretamente dependente das métricas fornecidas pelo sistema. Dentre estas métricas estão: a medida de tempo de reação oriunda do PVT; a curva de diâmetro da pupila (informadas em pixel ou valor absoluto) e os eventos de piscamento que são próprias da pupilometria.

Considerando-se que o equipamento desenvolvido é uma nova ferramenta com potencial para o estudo de privação de sono, é necessário que as métricas por ele fornecidas sejam avaliadas de forma criteriosa antes de aplicá-las na obtenção dos indicadores. Estas métricas são dependentes das características do equipamento e da metodologia do ensaio, que serão detalhadas a seguir para o PVT e para a pupilometria.

\section{Metodologia para obtenção da métrica do PVT}

A metodologia aplicada para obtenção da métrica Tempo de Reação (TR) do PVT, neste sistema, apresenta peculiaridades na sua forma que a diferencia dos demais ensaios de PVT. Entretanto, o ensaio segue o procedimento básico de um teste psicomotor constituído basicamente da apresentação de um estímulo visual e da monitoração da reação motora do sujeito. Neste sistema, o controle do experimento é determinado por quatro tempos distintos sendo estes configuráveis pelo usuário: Ensaio, Janela, Max_Reação e Leitura. O tempo Ensaio define a duração total da gravação que é dividida em seções denominadas trials. Um trial é iniciado com a estimulação visual gerada em um tempo aleatório de 0 a 8 segundos, sendo o limite superior deste intervalo configurado na variável Janela. Em seguida é monitorada a reação motora do sujeito, onde é 
registrado o intervalo de tempo percorrido entre a estimulação visual e a resposta do sujeito. A variável Max_Reação controla o máximo tempo em que o sistema mantém o estímulo presente (LEDs na vertical ligados). Um trial é finalizado com a apresentação em tela do respectivo tempo de reação ao estímulo, para que o sujeito acompanhe o seu desempenho. A variável Leitura determina o tempo de permanência na tela desta informação. Este procedimento irá se repetir até que seja atingido o tempo total da gravação.

A Figura 4 apresenta um intervalo de captura relativo ao início de um trial com os sinais envolvidos na avaliação do TR. O gráfico é constituído de três sinais: LED (linha pontilhada) indica o estado dos LEDs dispostos na vertical L3 e L4 (Figura 2a), nível lógico alto se ligados e nível lógico baixo se desligados, vale lembrar que os LEDs dispostos na horizontal (Figura 2a) atuam de forma inversa aos LEDs dispostos na vertical; Botão (linha contínua com tom cinza) permanece em nível lógico baixo enquanto o botão estiver pressionado; O sinal Início (linha contínua preta) é responsável pela indicação de um novo trial sendo sua sinalização feita por um pulso (nível alto) de $8,33 \mathrm{~ms}$ (Figura 4a). A partir deste pulso, num tempo aleatório entre 0 e 8 segundos, os LEDs dispostos na vertical que se encontram apagados (nível baixo sinal LED) são ligados gerando o estímulo visual (Figura 4b). O sinal Botão se mantém em nível lógico alto até que o sujeito responda ao estímulo pressionando o botão (Figura 4c). Ao detectar o botão pressionado, o software de monitoramento retira a estimulação com o apagamento dos LEDs dispostos na vertical (Figura 4d). O intervalo existente entre as descidas dos sinais Botão (Figura 4c) e LED (Figura 4d) é a latência do sistema e corresponde ao tempo entre dois quadros do filme. Este valor é dependente do número de FPS (frames per second) configurado para a coleta. $\mathrm{O}$ sinal Botão permanece em nível lógico baixo enquanto o botão estiver pressionado. $\mathrm{O}$ tempo entre a subida do sinal LED (Figura 4b) e a descida do sinal Botão (Figura 4c) determina a métrica tempo de reação (TR) relativa ao PVT e que neste caso foi de aproximadamente $400 \mathrm{~ms}$.

\section{Metodologia para obtenção das métricas da pupilometria}

Na obtenção das métricas pupilométricas cada quadro do filme passa pelo processamento de imagens constituído das etapas de segmentação e estimação. Diversas técnicas tradicionais como limiarização, ROI (region of interest), filtros morfológicos (erosão e dilatação) (Gonzalez e Woods, 1992), detecção de borda (Canny, 1986) e uma adaptação do Randomize Circle Detect (RCD) (Chen e Chung, 2001) foram aplicados para a estimação do centro, raio e abertura da pálpebra em relação à borda inferior da pupila (Figura 5) - que informa o quanto da pupila encontra-se exposta à luz. Caso seja definida a relação pixel $/ \mathrm{mm}$, antes da realização do ensaio, a partir de um padrão fixado sobre a pálpebra do sujeito (Figura 5), a estimação do diâmetro da pupila é realizada em valor absoluto.

A partir do diâmetro obtido quadro a quadro é gerado o sinal temporal base que passa por uma etapa de identificação de falhas de captura (Figura 6a) resultando no sinal corrigido (linha pontilhada).

Estas falhas são identificadas por meio do timestamp presente na imagem, sendo inserida uma amostra para cada quadro perdido (Figura 6a). A amplitude desta amostra é 2 vezes a média das amostras do sinal base cuja estimação é diferente de zero. O objetivo da atribuição deste valor para a amostra perdida é criar uma saturação positiva no sinal, diferenciando-a dos eventos de piscamento cuja amplitude da amostra recebe valor igual a zero

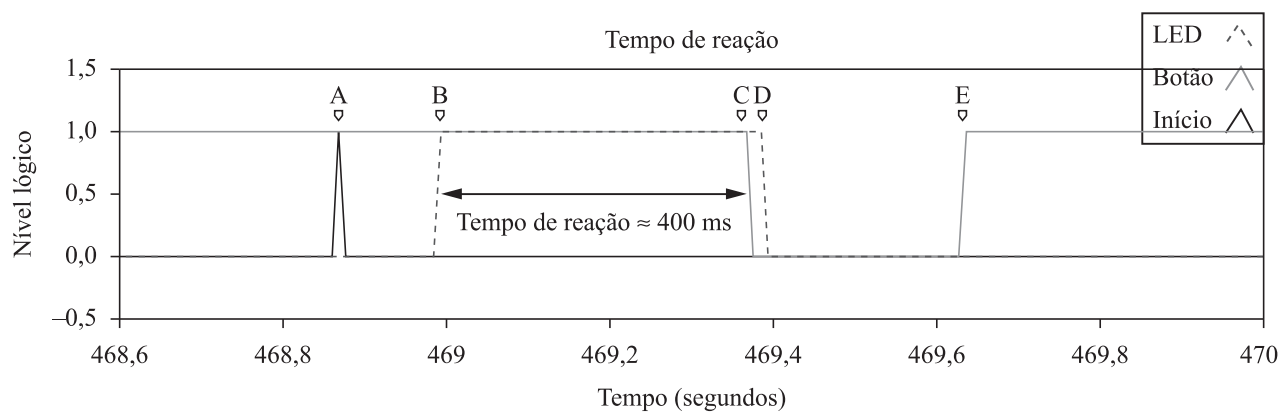

Figura 4. Sinais relativos a um trecho de captura. A amplitude dos sinais é definida em nível lógico por se tratar de estado ligado ou desligado. A) Sinaliza o início de um trial; B) Indica o início de uma estimulação (LEDs na vertical ligados); C) Momento em que o sujeito pressiona o botão; D) Retirada da estimulação pelo sistema (LEDs na horizontal ligados); E) Sujeito libera o botão.

Figure 4. Signs relative to a capture stretch. The signals' amplitude is defined as logical level (status ON or OFF). A) Points to the beginning of a trial; B) Indicates the beginning of a stimulation (LEDs in vertical position are ON); C) Moment in which the switch is pushed; D) Withdrawal of stimulation by the system (LEDs in horizontal position are ON); E) Subject releases the switch. 
(Figura 6b, c). No sinal corrigido é aplicada a remoção de artefatos onde os pontos pertencentes aos eventos de falhas de captura (Figura 6a) e aos piscamentos (Figura 6b, c) são substituídos por uma interpolação linear. Em seguida é aplicado um filtro Butterworth passa baixa de segunda ordem com frequência de corte de $2 \mathrm{~Hz}$ para obtenção do sinal filtrado (linha contínua) (Figura 6). Esta curva do diâmetro da pupila é a métrica da pupilometria que poderá ser aplicada no cálculo de indicadores de privação de sono.

A métrica de eventos de piscamento é obtida com base nas medidas de diâmetro da pupila (Figura 5). Para o caso de uma obstrução em que o algoritmo de detecção não seja capaz de identificar a pupila, o algoritmo de estimação associa o valor zero para o diâmetro da pupila. Desta forma, os quadros consecutivos cujos respectivos diâmetros apresentem valor zero, são considerados como um evento de piscamento. Embora o evento de piscamento seja considerado um artefato na obtenção de indicadores associados à oscilação da pupila, diversos estudos apontam-no como um potencial indicador de sonolência (Caffier et al., 2003; Malbouisson et al., 2010).

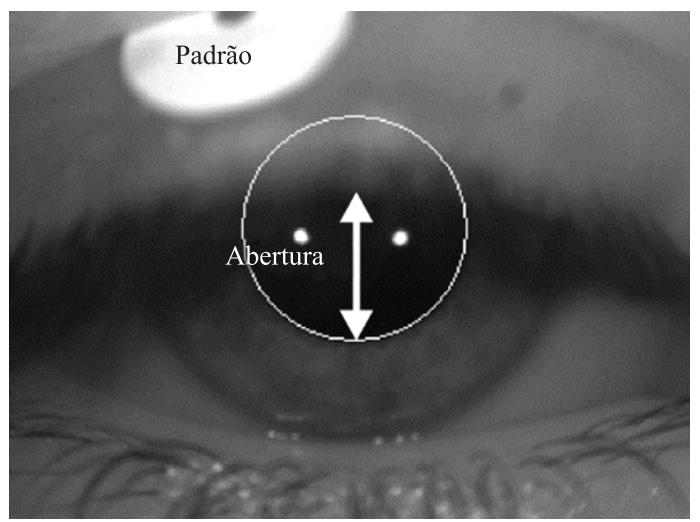

Figura 5. Estimações pupilométricas fornecidas pelo sistema: diâmetro da pupila e abertura de pálpebra. Padrão métrico utilizado como referência para estimações em valores absolutos.

Figure 5. Pupillometric stimulations provided by the system: pupil's diameter and eyelid's opening. Metric standard used as reference for estimates in absolute values.

\section{Avaliação das métricas}

Com objetivo de avaliar as métricas obtidas pelo sistema foi configurado um padrão de ensaio cuja duração da coleta é de 10 minutos, com 2 segundos para leitura do tempo de reação e com 5 segundos para o tempo máximo de reação. Esta configuração adotada para o ensaio é baseada em 'Basner e Dinges (2011).

Dos 19 sujeitos envolvidos no experimento, 3 não foram considerados devido a problemas como: câmera desalinhada em relação ao olho e longos períodos de olho fechado durante o processo de coleta que resultaram em imagens de baixa qualidade e consequentemente impróprias para o processamento. Para o grupo restante de 16 sujeitos foram processadas e avaliadas as métricas fornecidas pelo PVT (TR) e pela pupilometria (diâmetro de pupila e eventos de piscamento) nos ensaios realizados após uma noite de sono o que caracterizaria o estado de alerta.

Além da avaliação das métricas, um estudo adicional foi realizado com o objetivo de demonstrar o diferencial deste equipamento. A partir da composição das métricas TR (PVT) e curva de diâmetro da pupila (pupilometria) é possível fornecer um indicador de lapsos diferenciado dos demais equipamentos de PVT. Originalmente o indicador de lapsos do PVT registra o somatório dos eventos de lapso, que é caracterizado por respostas cujo TR $>500 \mathrm{~ms}$ ou por um falso início (FI) que é resultado de um falsa detecção (FD) - TR $<100 \mathrm{~ms}$ - ou por uma falsa resposta (FR) - resposta sem estímulo (Basner e Dinges, 2011). O evento de lapso associado à métrica de curva de diâmetro da pupila possibilita distinguir se o sujeito estava com o olho aberto ou fechado durante o evento de lapso. Esta distinção, segundo Anderson et al. (2010), permite identificar o grau de engajamento do sujeito na tarefa executada.

Além das métricas relacionadas ao indicador fisiológico ou comportamental, serão avaliadas algumas métricas de funcionamento do sistema, como: o número de trials gerados pelo sistema ao longo do ensaio, a metodologia de estimulação visual adotada e resolução das medidas pupilométricas.

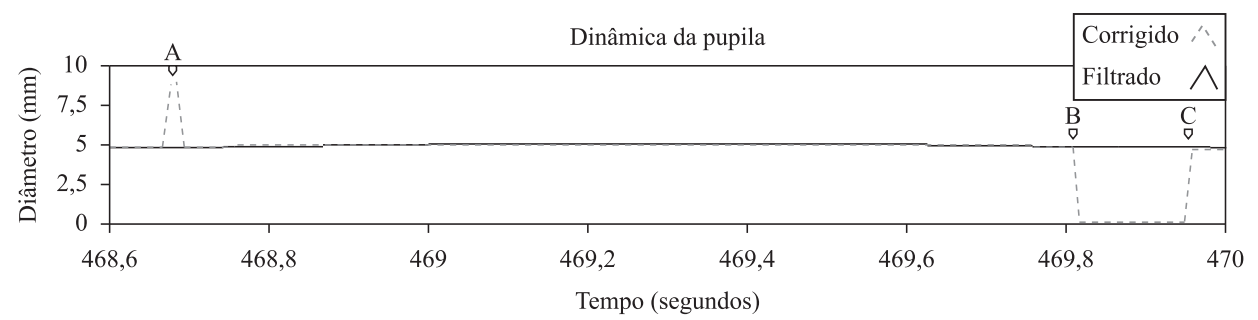

Figura 6. A) Sinaliza uma falha de captura, B) início e C) fim de um evento de piscamento.

Figure 6. A) Points to a capture failure, B) beginning and C) end of blink event. 


\section{Resultados}

Na escala PANAS-X (Positive and Negative Affect Schedule - Expanded Form) os indivíduos obtiveram uma média $(7,3 \pm 1,8)$, abaixo do seu valor central (10), sendo que apenas o indivíduo 16 superou, em um ponto, este limite (Tabela 1).

Na métrica exclusiva do PVT, o tempo médio de reação (TR Médio) foi de 374,6 $\pm 47,1$ ms, o que representa uma variabilidade próxima de $12 \%$ em relação à média.

Nas métricas geradas exclusivamente pela pupilometria a taxa de piscamento por minuto apresentou maior variabilidade $(\approx 74 \%)$ - com média de $7,68 \pm 5,96$ eventos/min - do que a do diâmetro médio da pupila (18\%) - com média de 4,66 $\pm 0,86 \mathrm{~mm}$.

No indicador padrão de lapsos que é oriundo da métrica TR do PVT os falsos inícios são basicamente determinados por falhas de resposta (FR), ocorrendo somente uma falha de detecção (FD) nos sujeitos 3 e 7 (Tabela 1). Na integração com a métrica de diâmetro da pupila identifica-se um predomínio de lapsos de olho aberto (LOA), ficando os lapsos de olho fechado (LOF) com percentual menor que $10 \%$ do total de lapsos.

Com relação às métricas exclusivas do sistema o número médio de trials foi de $81,38 \pm 7,44$ para os ensaios de PVT com duração de 10 minutos (Tabela 1).
A Figura 7 (Tempo de Reação) ilustra um caso típico de lapso (Tempo de Reação maior que $500 \mathrm{~ms}$ ) ocorrido durante o ensaio de um voluntário. Juntamente com a curva do diâmetro da pupila (Figura 7 - Dinâmica da pupila) é possível identificar que o respectivo evento é um lapso com olho aberto (LOA).

As Figura 8a, b mostram os resultados das segmentações das imagens das respectivas Figura 3a, b com um limiar de nível de cinza igual a 175. A troca de posição dos pontos brancos, da horizontal para vertical, é a mudança que ocorre entre as imagens dos dois quadros consecutivos no instante que é iniciada a estimulação visual, ou seja, é a diferença visual que será percebida, na tela do computador, pelo sujeito. $\mathrm{Na}$ Figura $8 \mathrm{a}$ os pontos brancos têm 44 e 52 pixels, que correspondem respectivamente a 0,057 e $0,068 \%$ dos 76800 pixels da imagem. Já na Figura 8b, os pontos brancos têm áreas de 48 e 49 pixels que correspondem respectivamente a 0,062 e $0,064 \%$.

Nos ensaios realizados, utilizou-se a taxa máxima de amostragem de $120 \mathrm{FPS}(\mathrm{T}=8,33 \mathrm{~ms}$ ) que permite uma resolução espacial máxima de $320 \times 240 \mathrm{em}$ 256 níveis de cinza. Nesta configuração a resolução das medidas pupilométricas foi próxima de $0,07 \mathrm{~mm}$.

Tabela 1. Métricas do PVT e da pupilometria e do indicador de lapsos obtidas em sujeitos após uma noite sono (estado de alerta) em ensaios com duração de 10 minutos.

Table 1. PVT and pupillometry measurements and lapses indicator obtained from subjects after a sleeping night in alert state, during 10 minutes trials.

\begin{tabular}{|c|c|c|c|c|c|c|c|c|c|}
\hline \multirow[b]{2}{*}{ Sujeito } & \multicolumn{4}{|c|}{ PVT } & \multicolumn{2}{|c|}{ PVT \& pupilometria } & \multicolumn{2}{|c|}{ Pupilometria } & \multirow{2}{*}{$\begin{array}{c}\text { PANAS-X } \\
(1-20)\end{array}$} \\
\hline & Trials & FR & FD & $\begin{array}{c}\text { TR } \\
\text { médio } \\
\text { (ms) }\end{array}$ & LOA & LOF & $\begin{array}{c}\text { Diâmetro } \\
\text { média } \\
(\mathrm{mm})\end{array}$ & $\begin{array}{c}\text { Pisca } \\
\text { taxa } \\
\text { (minutes) }\end{array}$ & \\
\hline 1 & 82 & 2 & 0 & 365,8 & 2 & 0 & 3,53 & 4,6 & 7 \\
\hline 2 & 81 & 0 & 0 & 394,4 & 1 & 2 & 5,93 & 9,0 & 7 \\
\hline 3 & 90 & 1 & 1 & 315,0 & 0 & 0 & 4,69 & 7,3 & 8 \\
\hline 4 & 93 & 4 & 0 & 382,1 & 1 & 0 & 4,80 & 1,6 & 8 \\
\hline 5 & 85 & 1 & 0 & 309,4 & 1 & 0 & 5,19 & 6,5 & 4 \\
\hline 6 & 71 & 1 & 0 & 440,0 & 16 & 1 & 5,86 & 10,8 & 6 \\
\hline 7 & 85 & 0 & 1 & 363,3 & 0 & 0 & 6,22 & 3,9 & 4 \\
\hline 8 & 87 & 0 & 0 & 342,4 & 2 & 0 & 4,50 & 6,6 & 6 \\
\hline 9 & 84 & 0 & 0 & 405,0 & 1 & 0 & 4,45 & 5,5 & 7 \\
\hline 10 & 84 & 1 & 0 & 311,2 & 1 & 0 & 5,18 & 3,3 & 7 \\
\hline 11 & 74 & 4 & 0 & 390,0 & 8 & 0 & 4,89 & 8,0 & 10 \\
\hline 12 & 70 & 7 & 0 & 424,6 & 9 & 1 & 3,65 & 19,5 & 7 \\
\hline 13 & 82 & 0 & 0 & 472,4 & 4 & 0 & 3,44 & 2,9 & 9 \\
\hline 14 & 67 & 3 & 0 & 388,1 & 10 & 2 & 3,99 & 23,4 & 8 \\
\hline 15 & 88 & 2 & 0 & 332,2 & 2 & 0 & 4,16 & 2,7 & 7 \\
\hline 16 & 79 & 2 & 0 & 357,3 & 1 & 0 & 4,04 & 7,3 & 11 \\
\hline Média & 81,38 & 1,750 & 0,125 & 374,6 & 3,69 & 0,38 & 4,66 & 7,68 & 7,3 \\
\hline DP & 7,44 & 1,949 & 0,342 & 47,1 & 4,60 & 0,72 & 0,86 & 5,96 & 1,8 \\
\hline
\end{tabular}




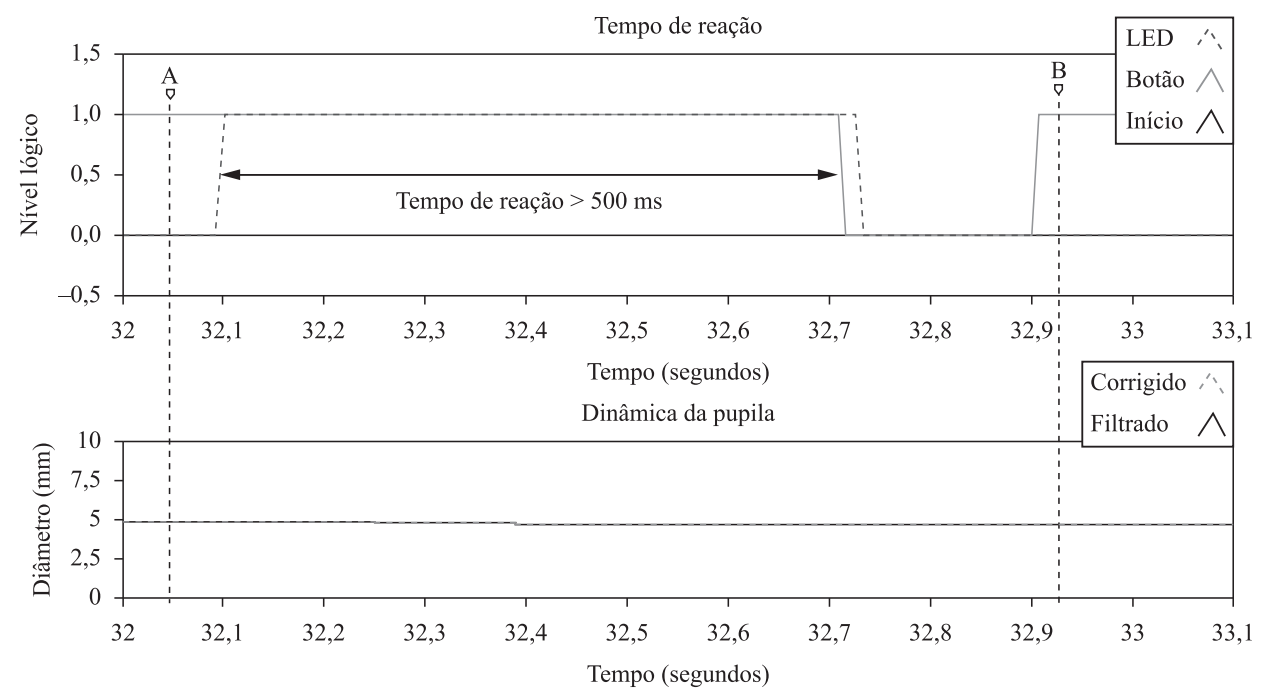

Figura 7. Exemplo típico de um lapso (LOA), que é caracterizado por um tempo de reação $>500 \mathrm{~ms}$ (Tempo de Reação). O gráfico "Dinâmica da pupila" mostra que, durante o intervalo delimitado pelas linhas verticais A e B, o olho permaneceu aberto, o que torna possível a discriminação entre lapsos com olho aberto e fechado.

Figure 7. Typical example of an eyes open lapse, characterized by a reaction time $>500 \mathrm{~ms}$ (Reaction Time). The chart "Pupil's Dynamics" shows that, during the interval set out by the vertical lines $A$ and $B$, the eye was kept open, allowing to distinguish between eyes open and eyes closed lapses.
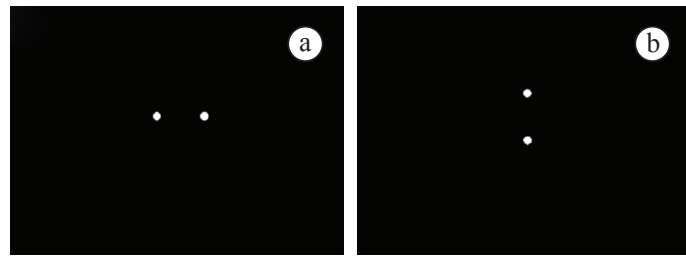

Figura 8. Resultado da limiarização com um nível de cinza de 175 aplicado sobre a a) Figura $5 \mathrm{~b}$ e b) Figura $5 \mathrm{c}$.

Figure 8. Result of thresholding with a gray level of 175 applied over a) Figure $5 b$ and b) Figure $5 c$.

\section{Discussão}

A escala PANAS-X, utilizada como balizadora do nível de alerta dos indivíduos envolvidos no experimento mostrou que todos os indivíduos não se apresentavam sonolentos, valores menores a 10 (Watson e Clark, 1994). Desta forma, todas as métricas a seguir serão avaliadas tendo como referência dados apresentados na literatura para sujeitos sem privação do sono.

O tempo de reação (TR) médio obtido nos ensaios ficou próximo de $350 \mathrm{~ms}$, i.e., em torno de $100 \mathrm{~ms}$ acima do reportado na literatura (Basner e Dinges, 2011; Kim et al., 2007; Lee et al., 2010). Trabalhos anteriores mostraram que diversos são os elementos que interferem no tempo de reação. Em 2011, Ratcliff e Van Dongen mostraram que a variação no tempo de reação está associada não só na metodologia do ensaio, mas também nas características do padrão de estimulação tais como brilho, tamanho e forma. Já
Reimers e Stewart (2008) mostram a relação do tempo de reação com o hardware utilizado na construção do equipamento do PVT. Entretanto, concluíram que se as diferenças intrínsecas dos equipamentos PVT são conhecidas e levadas em conta no processo de obtenção dos indicadores, não existe nenhum fator impeditivo para aplicação de tais equipamentos nos ensaios de PVT. A referência utilizada neste trabalho, de $\mathrm{TR} \approx 250 \mathrm{~ms}$, foi obtida em uma publicação de Basner e Dinges (2011), onde os autores buscaram estabelecer um padrão para ensaio de PVT objetivando a comparação sistematizada entre os diversos ensaios que envolvam a aplicação do PVT. Entretanto, nos respectivos experimentos não foi apresentada uma descrição clara do processo de estimulação. Os autores descreveram que a estimulação visual foi realizada utilizando o monitor de um computador, onde é apresentado um retângulo de fundo vermelho. A estimulação está associada ao surgimento de um contador, com dígitos na cor amarela, que realiza uma contagem em ordem crescente. O tempo de reação é calculado tendo como referência o momento em que o sujeito pressiona um botão que interrompe a contagem. Não existe ao longo da descrição do procedimento de estimulação qual a taxa de ocupação deste retângulo no monitor e tão pouco a forma e o tamanho dos dígitos do contador. Com relação à descrição do equipamento utilizado nos experimentos os autores mostraram uma preocupação com relação à plataforma utilizada, mas 
a descreve vagamente, não sendo possível determinar se é um sistema de tempo real.

Segundo nosso conhecimento, o sistema desenvolvido neste trabalho utiliza uma metodologia de estimulação distinta em relação aos demais equipamentos de PVT. Embora exista a dependência da estimulação para o cálculo do tempo de reação, é fundamental minimizar a indução de oscilação pupilar (constrição e dilatação) causada pela estimulação visual, pois compromete as medidas pupilométricas. Desta forma, o padrão de estimulação utilizado neste sistema (Figura 3a, b), que corresponde aos pontos brancos relativos ao reflexo da córnea às fontes de IR, mantém-se praticamente inalterado à emissão de luz pelo monitor do computador. As Figura 8a, b são resultantes do processo de aplicação do limiar de nível de cinza 175 nas Figura 3a, b, respectivamente. Este procedimento foi realizado para ilustrar a variação que ocorre entre as duas imagens consecutivas, antes e após o evento de estimulação. $\mathrm{O}$ resultado da troca das fontes de iluminação provoca apenas o reposicionamento (horizontal para vertical) dos pontos relativo ao reflexo corneal na imagem, mantendo praticamente inalterada a emissão de luz pelo monitor. Já com relação ao padrão de estimulação este ocupa apenas uma região de $0,2 \%$ da imagem, produzindo uma pequena variabilidade de informação entre os quadros responsáveis pela estimulação. Desta forma, acreditamos que o tempo de reação de $100 \mathrm{~ms}$ acima do que o obtido por Basner e Dinges (2011) pode ser devido a maior dificuldade de sua identificação pelo sujeito. Em contra partida é uma técnica alternativa para sistemas, como o aqui proposto, que são sensíveis à modulação pupilar.

Em 1990, Spector informou que o diâmetro da pupila em adultos varia de 2 a $4 \mathrm{~mm}$ no claro e de 4 a $8 \mathrm{~mm}$ no escuro, entretanto as quantificações dos níveis de claro e escuro não foram especificadas. Em 2006, Bremner e Smith, determinaram a relação expected dark diameter $\mathrm{DDe}=8,283-(0,043 \times \mathrm{A}) \mathrm{mm}$, onde A é a idade do voluntário, em ambiente escotópico, mas novamente sem apresentar valor quantitativo desta medida. Já em 2011, Bradley et al. realizaram experimentos com indivíduos saudáveis, que passaram por uma fase de adaptação ao escuro (1 lux) e os resultados encontrados para faixa de 20 a 29 anos foi de $7,33 \mathrm{~mm}(5,7$ a $8,8 \mathrm{~mm})$ para $\mathrm{n}=66$ voluntários. A média entre todas as faixas etárias de 18 e 80 anos variou de $6,85(5,6$ a 7,5$) \mathrm{mm}$ a $4,85(4,1$ a 5,3$) \mathrm{mm}$, respectivamente. Entretanto, é importante ressaltar que estas medidas foram realizadas entre 14 e 20 horas que podem conter alterações circadianas que interfiram no tamanho do diâmetro da pupila. Embora a média do diâmetro da pupila encontrada no presente experimento $(4,6 \mathrm{~mm})$ esteja próxima das faixas relatadas nos experimentos anteriores, ela encontra-se abaixo do especificado para média de idade dos 16 voluntários. Estes valores podem estar relacionados com inexistência de adaptação ao ambiente antes da execução do ensaio e principalmente com o nível de iluminância $(\approx 20$ lux $)$ no qual permaneceram os voluntários durante os ensaios. A iluminância de 20 lux foi adotada para minimizar os efeitos da luz visível na pupila.

A taxa de piscamento é utilizada como um indicador tanto em estudos de privação do sono como em testes psicofísicos envolvendo estimulação visual (Stern et al., 1984). Entretanto, Al-Abdulmunem e Briggs (1999) demonstraram que uma série de fatores influencia na taxa de piscamento espontâneo. Em 1997, Bentivoglio et al. mostraram que em descanso a taxa de piscamento por minuto é de 17 , subindo para 26 numa conversa e caindo para 4,5 em uma leitura. Já em 2001, Wong et al. mostraram por meio de comparação entre uma conversa e uma tarefa visual de leitura ou a execução de cirurgia uma queda na taxa de piscamento/ minuto de 16,69 para 4,75 eventos. Considerando que a tarefa visual executada em nosso experimento não demanda um esforço cognitivo envolvido numa leitura, a taxa média obtida em nossos experimentos de 7,68 reflete o comportamento característico de uma simples tarefa visual.

Quanto ao indicador de lapsos (LOA + LOF + FD + FR) a sua média foi de 5,95 para ensaio de 10 minutos, que é próxima à obtida (5 lapsos) por Basner e Dinges (2011) para uma população (homens e mulheres) com idade média de 30,5 \pm 7,3 anos.

Considerando o tempo máximo de reação (5 segundos), o tempo de duração do trial pode variar entre 2 e 15 segundos. Durante 10 minutos de ensaio (600 segundos), totalizaria um mínimo de 40 e o máximo de 300 trials. Para todos os ensaios o sistema funcionou corretamente, pois o número trials ficou dentro desta faixa, sendo que a média encontra-se 2 vezes acima do limite inferior e 3,7 vezes abaixo do limite superior. Observando os extremos (inferior/superior) obtidos no número de trials e tempo médio de reação (Tabela 1) tem no sujeito 5 o menor tempo médio de reação, entretanto ele não apresenta o maior número de trials, que ocorre no sujeito 4. Já o sujeito 14 que apresentou o menor número de trials não detém a maior média de tempo de reação, que é do sujeito 13. Estes resultados mostram a inexistência de uma relação inversamente proporcional entre o TR e o número de trials. Onde se conclui que o estímulo aleatório atua de forma significativa evitando assim a possibilidade de uma tendência temporal da estimulação. 
Esta nova ferramenta integra, em um único dispositivo, o PVT e a pupilometria, possibilitando a distinção de lapsos com o olho aberto (Figura 7) e fechado, que é um importante marcador do grau de engajamento do sujeito na tarefa realizada (Anderson et al., 2010).

Devido ao seu processamento offline, o dispositivo atualmente não fornece a informação do diâmetro em tempo real. Entretanto, é possível distinguir por meio do filme gravado se as estimações de pupila com diâmetro zero correspondem realmente a eventos de piscamento. Isto não é possível em diversos eyetracks e em sistemas tais como descrito em Bernhardt et al. (1996) que fornecem medidas de diâmetro iguais a zero tanto para uma falha de captura e um evento de piscamento.

A resolução das medidas pupilométricas obtida pelo sistema está dentro da faixa 0,01 a $0,1 \mathrm{~mm}$ encontradas nas especificações de pupilômetros disponíveis no mercado. Ela pode atingir até $0,03 \mathrm{~mm}$ desde que a taxa de amostragem seja reduzida para $60 \mathrm{~Hz}$. Com sua resolução temporal máxima de $8,33 \mathrm{~ms}$ e considerando que a principal medida efetuada neste experimento foi o evento de lapso ( $>500 \mathrm{~ms})$, o erro máximo fica limitado a um quadro $(\approx 1,7 \%)$.

$\mathrm{O}$ sincronismo entre a aquisição de dados do sistema (captura da imagem e sinal de resposta do voluntário) e a geração da estimulação ocorre na própria câmera, assim, não é necessário nenhum sinal adicional para garantir a sincronização entre eles.

Além de se fundamentar em técnicas não invasivas, é importante ressaltar que a portabilidade e a praticidade de operação do sistema desenvolvido viabilizam sua aplicação além dos limites de um laboratório. Dispensa a necessidade de um corpo técnico para sua operação e demanda um curto tempo de preparação do sujeito $(\approx 2$ minutos) que é fundamental para aplicações em larga escala nas atividades clínicas e cotidianas (Kirsch e Adusumilli, 2009). O alarme sonoro característico do PVT auxilia na coleta de dados pupilométricos evitando assim longos trechos de olho fechado. Também minimiza/elimina a necessidade do aplicador despertar o sujeito nestas situações, que são frequentes nos ensaios de pupilometria para sujeitos privados do sono.

Por outro lado, um fator limitante deste sistema é sua abordagem monocular, pois se aplica somente a sujeitos que não apresentem disfunções visuais restritivas a sua utilização.

Estudos futuros avaliando a influência do nível de iluminância no diâmetro da pupila poderão identificar a viabilidade de aplicação do sistema para ensaios com maior presença de luz ambiente.

Estamos estabelecendo novos procedimentos de ensaios para avaliação da privação do sono que garantam condições distintas (com e sem privação de sono). Esta abordagem permite a aplicação de testes pareados para levantar indicadores de privação de sono fundamentados no hippus (oscilação da pupila), miosis (constrição da pupila) e na dinâmica palpebral que são oriundos da pupilometria e no tempo de reação oriundo do PVT. Com os indicares obtidos buscaremos estabelecer limiares que nos permitam identificar sujeitos privados de sono.

\section{Conclusão}

Com este trabalho foi possível mostrar que o sistema é eficiente na obtenção simultânea das diversas métricas oriundas do PVT e da pupilometria, garantido que isto ocorra para as mesmas condições psicofisiológicas do sujeito. Os resultados das métricas e o indicador de lapsos se mostraram em conformidade com a literatura, apresentando algumas variações inerentes da abordagem de hardware utilizada no PVT. Entretanto, para o estudo de privação de sono um novo protocolo é necessário. Neste caso, o sujeito será privado de sono de forma assistida por um período de 36 horas, onde serão realizadas coletas periódicas, que possibilitarão acompanhar as variações circadianas e a realização de testes pareados.

Embora o sistema tenha sido originalmente projetado para pesquisas de sonolência, testes pupilométricos e psicofísicos apresentam uma extensa lista de aplicações: Alzheimer (Fotiou et al., 2009), alcoolismo (Rubin, 1980), ansiedade/desordem do pânico (Kojima et al., 2004), autismo (Fan et al., 2009), depressão (Siegle et al., 2004), diabetes (Ferrari et al., 2010), esclerose múltipla (Frauscher et al., 2005), Parkinson (Hori et al., 2008) e esquizofrenia (Granholm e Verney, 2004).

Vale ressaltar que o conhecimento adquirido no desenvolvimento do sistema nos capacita a agregar novas funcionalidades (software) e possíveis expansões (hardware) para atender a sua ampla aplicabilidade, assim como a experimentos futuros que se baseiem em novos paradigmas, inclusive com sinais eletrofisiológicos.

\section{Agradecimentos}

Este trabalho teve apoio financeiro da Fundação de Amparo à Pesquisa do Estado de Minas Gerais - FAPEMIG, Conselho Nacional de Desenvolvimento Científico e Tecnológico - CNPq, da Coordenação de Aperfeiçoamento de Pessoal de Nível Superior - CAPES e da FAPESP (processo $n^{\circ} 2011 / 01286-0$ ). 


\section{Referências}

Al-Abdulmunem MA, Briggs ST. Spontaneous blink rate of a normal population sample. ICLC 1999; 26.

Anderson C, Wales AW, Horne JA. PVT lapses differ according to eyes open, closed, or looking away. Sleep. 2010; 33:197-204. PMid:20175403 PMCid:2817906.

Basner M, Dinges DF. Maximizing sensitivity of the psychomotor vigilance test (PVT) to sleep loss. Sleep. 2011; 34:581-91. PMid:21532951 PMCid:3079937.

Beck AT, Ward CH, Mendelson M, Mock J, Erbaugh J. An inventory for measuring depression. Archives of General Psychiatry. 1961; 4:561-71. PMid:13688369. http://dx.doi. org/10.1001/archpsyc.1961.01710120031004

Benedito-Silva AA, Menna-Barreto L, Marques N, Tenreiro $\mathrm{S}$. A self-assessment questionnaire for the determination of morningness-eveningness types in Brazil. Progress in Clinical and Biological Research. 1990: 89-98. PMid:2217379.

Bentivoglio AR, Bressman SB, Cassetta E, Carretta D, Tonali P, Albanese A. Analysis of blink rate patterns in normal subjects. Movement Disorders. 1997; 12:1028-34. PMid:9399231. http://dx.doi.org/10.1002/mds.870120629

Bernhardt PC, Dabbs JMD, Riad JK. Pupillometry system for use in social psychology. Behavior Research Methods, Instruments. \& Computers. 1996; 28:61-6. http://dx.doi. org/10.3758/BF03203637

Bitsios P, Schiza SE, Giakoumaki SG, Savidou K, Alegakis AK, Siafakas N. Pupil miosis within 5 minutes in darkness is a valid and sensitive quantitative measure of alertness: application in daytime sleepiness associated with sleep apnea. Sleep. 2006; 29:1482-8. PMid:17162996.

Bradley JC, Bentley KC, Mughal AI, Bodhireddy H, Brown SM. Dark-adapted pupil diameter as a function of age measured with the NeurOptics pupillometer. Journal of Refractive Surgery. 2011; 27:202-7. PMid:20506961.

Bremner F, Smith S. Pupil findings in a consecutive series of 150 patients with generalised autonomic neuropathy. Journal of Neurology, Neurosurgery \& Psychiatry. 2006; 77:1163-8. PMid:16754691 PMCid:2077530. http://dx.doi.org/10.1136/ jnnp.2006.092833

Caffier PP, Erdmann U, Ullsperger P. Experimental evaluation of eye-blink parameters as a drowsiness measure. European Journal of Applied Physiology. 2003; 89:319-25. PMid:12736840. http://dx.doi.org/10.1007/s00421-0030807-5

Caffier PP, Erdmann U, Ullsperger P. The spontaneous eye-blink as sleepiness indicator in patients with obstructive sleep apnoea syndrome-a pilot study. Sleep Medicine. 2005; 6:155-62. PMid:15716219. http://dx.doi. org/10.1016/j.sleep.2004.11.013

Canny J. A computational approach to edge detection. IEEE Transactions on Pattern Analysis and Machine Intelligence. 1986; 8: 679-98. PMid:21869365. http://dx.doi. org/10.1109/TPAMI.1986.4767851

Carskadon MA, Dement WC, Mitler MM, Roth T, Westbrook PR, Keenan S. Guidelines for the multiple sleep latency test (MSLT): A standard measure of sleepiness. Sleep. 1986; 9:519-24. PMid:3809866.
Chen T-C, Chung K-L. An efficient randomized algorithm for detecting circles. Computer Vision and Image Understanding. 2001; 83:172-91. http://dx.doi.org/10.1006/ cviu. 2001.0923

Center for Narcolepsy, Sleep and Health Research - CNSHR Illinois, 2012

Curcio G, Casagrande M, Bertini M. Sleepiness: Evaluating and quantifying methods. International Journal of Psychophysiology. 2001; 41:251-63. http://dx.doi. org/10.1016/S0167-8760(01)00138-6

Dinges DF, Powell JW. Microcomputer analyses of performance on a portable, simple visual RT task during sustained operations. Behavior Research methods, Instruments \& Computers. 1985; 16:652-5. http://dx.doi.org/10.3758/ BF03200977

Fan X, Miles JH, Takahashi N, Yao G. Abnormal transient pupillary light reflex in individuals with autism spectrum disorders. Journal of Autism and Developmental Disorders. 2009; 39:1499-508. PMid:19499319. http:// dx.doi.org/10.1007/s10803-009-0767-7

Ferrari GL, Marques JL, Gandhi RA, Heller SR, Schneider FK, Tesfaye S, Gamba HR. Using dynamic pupillometry as a simple screening tool to detect autonomic neuropathy in patients with diabetes: a pilot study. BioMedical Engineering OnLine. 2010; 9:26. PMid:20565794 PMCid:2893537. http://dx.doi.org/10.1186/1475-925X-9-26

Fotiou DF, Stergiou V, Tsiptsios D, Lithari C, Nakou M, Karlov A. Cholinergic deficiency in Alzheimer's and Parkinson's disease: evaluation with pupillometry. International Journal of Psychophysiology. 2009; 73:143-9. PMid:19414041. http://dx.doi.org/10.1016/j.ijpsycho.2009.01.011

Franzen PL, Siegle GJ, Buysse DJ. Relationships between affect, vigilance, and sleepiness following sleep deprivation. Journal of Sleep Research. 2008; 17:34-41. PMid:18275553 PMCid:3107826. http://dx.doi.org/10.1111/ j.1365-2869.2008.00635.x

Frauscher B, Egg R, Brandauer E, Ulmer H, Berger T, Poewe W, Hogl B. Daytime sleepiness is not increased in mild to moderate multiple sclerosis: pupillographic study. Sleep Medicine. 2005; 6:543-7. PMid:16084765. http:// dx.doi.org/10.1016/j.sleep.2005.05.001

Gonzalez RCE, Woods RE. Digital image processing. Addison-Wesley Publishing Company; 1992.

Gorenstein C, Pompeia S, Andrade L. Scores of Brazilian university students on the Beck Depression and the State Trait Anxiety Inventories. Psychological Reports. 1995; 77:635-41. PMid:8559894. http://dx.doi.org/10.2466/pr0.1995.77.2.635

Granholm E, Verney SP. Pupillary responses and attentional allocation problems on the backward masking task in schizophrenia. International Journal of Psychophysiology. 2004; 52:37-51. PMid:15003371. http:// dx.doi.org/10.1016/j.ijpsycho.2003.12.004

Hoge EA, Marques L, Wechsler RS, Lasky AK, Delong HR, Jacoby RJ, Worthington JJ, Pollack MH, Simon NM. The role of anxiety sensitivity in sleep disturbance in panic disorder. Journal of Anxiety Disorders. 2011; 25:536-8. PMid:21277737. http://dx.doi.org/10.1016/j. janxdis.2010.12.008 
Hori N, Takamori M, Hirayama M, Watanabe H, Nakamura T, Yamashita F, Ito H, Mabuchi N, Sobue G. Pupillary supersensitivity and visual disturbance in Parkinson's disease. Clinical Autonomic Research. 2008; 18:20-7. PMid:18265941. http://dx.doi.org/10.1007/s10286-008-0453-4

Horne JA, Ostberg O. A self-assessment questionnaire to determine morningness-eveningness in human circadian rhythms. International Journal of Chronobiology. 1976; 4: 97-110. PMid:1027738.

International Commission on Non-Ionizing Radiation Protection - ICNIRP ICoN-IRP. Guidelines on limits of exposure to broad-band incoherent optical radiation. Health Physics Societ. 1997:539-54.

Johns MW. A new method for measuring daytime sleepiness: the Epworth sleepiness scale. Sleep. 1991; 14:540-5. PMid:1798888.

Kim H, Dinges DF, Young T. Sleep-disordered breathing and psychomotor vigilance in a community-based sample. Sleep. 2007; 30:1309-16. PMid:17969464 PMCid:2266275.

Kirsch D, Adusumilli J. Multiple sleep latency test and maintenance of walkefulness test. Sleep Medicine Clinics. 2009; 385-92. http://dx.doi.org/10.1016/j. jsmc.2009.04.006

Kojima M, Shioiri T, Hosoki T, Kitamura H, Bando T, Someya T. Pupillary light reflex in panic disorder. A trial using audiovisual stimulation. European Archives of Psychiatry and Clinical. 2004; 254:242-4.

Lee IS, Bardwell WA, Ancoli-Israel S, Dimsdale JE. Number of lapses during the psychomotor vigilance task as an objective measure of fatigue. Journal of Clinical Sleep Medicine. 2010; 6:163-8. PMid:20411694 PMCid:2854704.

Liang JA, Sun LM, Muo CH, Sung FC, Chang SN, Kao $\mathrm{CH}$. Non-apnea sleep disorders will increase subsequent liver cancer risk - A nationwide population-based cohort study. Sleep Medicine. 2012. PMid:22503943. http://dx.doi. org/10.1016/j.sleep.2012.02.005

Liu D, Pang Z, Lloyd SR. A neural network method for detection of obstructive sleep apnea and narcolepsy based on pupil size and EEG. IEEE Transactions on Neural Networks. 2008; 19:308-18. PMid:18269961. http://dx.doi. org/10.1109/TNN.2007.908634

Malbouisson JM, Messias A, Garcia DM, CechettiSde P, Barbosa JC, Cruz AA. Modeling upper eyelid kinematics during spontaneous and reflex blinks. Journal of Neuroscience Methods. 2010; 191:119-25. PMid:20547184. http://dx.doi. org/10.1016/j.jneumeth.2010.06.006

McLaren JW, Hauri PJ, Lin SC, Harris CD. Pupillometry in clinically sleepy patients. Sleep Medicine. 2002; 3:347-52. http://dx.doi.org/10.1016/S1389-9457(02)00017-5

Patil SG, Gale TJ, Stack CR. Design of novel assessment techniques for opioid dependent patients. Conference Proceedings IEEE Engineering in Medicine and Biology Society. 2007; 2007:3737-40.

Porcu S, Casagrande M, Ferrara M, Bellatreccia A. Sleep and alertness during alternating monophasic and polyphasic rest-activity cycles. International Journal of
Neuroscience. 1998; 95:43-50. PMid:9845015. http://dx.doi. org/10.3109/00207459809000648

Ratcliff R, Van Dongen HP. Diffusion model for one-choice reaction-time tasks and the cognitive effects of sleep deprivation. Proceedings of the National Academy of Sciences of the United States of America. 2011; 108:11285-90. PMid:21690336 PMCid:3131321. http://dx.doi.org/10.1073/ pnas. 1100483108

Raven JC, Raven J, Court JH. Manual - Matrizes progressivas coloridas de Raven. São Paulo: Casa do Psicologo; 1988.

Reimers S, Stewart N. Using Adobe Flash Lite on mobile phones for psychological research: Reaction time measurement reliability and interdevice variability. Behavior Research Methods. 2008; 40:1170-6. PMid:19001409. http:// dx.doi.org/10.3758/BRM.40.4.1170

Rubin LS. Pupillometric studies of alcoholism. International Journal of Neuroscience. 1980; 11:301-8. PMid:7451037. http://dx.doi.org/10.3109/00207458009147594

Siegle GJ, Steinhauer SR, Thase ME. Pupillary assessment and computational modeling of the Stroop task in depression. International Journal of Psychophysiology. 2004; 52:6376. PMid:15003373. http://dx.doi.org/10.1016/j. ijpsycho.2003.12.010

Smolensky MH, Di Milia L, Ohayon MM, Philip P. Sleep disorders, medical conditions, and road accident risk. Accident Analysis and Prevention. 2010; 43:533-48. PMid:21130215. http://dx.doi.org/10.1016/j.aap.2009.12.004

Spector RH. The pupils. In: Walker HK, Hall WD, Hurst JW, editors. Clinical methods: The history, physical, and laboratory examinations. Boston; 1990.

Spielberger CD, Gorsuch RL, Lushene RE. Manual for the State-Trait Anxiety Inventory. Palo Alto: Consulting Psychologists Press; 1970.

Stern JA, Boyer D, Schroeder DJ. Blink rate as a measure of fatigue: A review. Washington: Office of Aviation Medicine Federal Aviation Administration; 1994.

Stern JA, Walrath LC, Goldstein R. The endogenous eyeblink. Psychophysiology. 1984; 21:22-33. PMid:6701241. http:// dx.doi.org/10.1111/j.1469-8986.1984.tb02312.x

Telles SCL, Corrêa ÉA, Caversan BL, MattosJdM, Alves RSC. Significado Clínico da Actigrafia. Revista Neurociências. 2011; 19:53-161.

Watson D, Clark LA. THE PANAS-X Manual for the positive and negative affect schedule - Expanded Form. The University of Iowa; 1994.

Wilhelm BJ, Widmann A, Durst W, Heine C, Otto G. Objective and quantitative analysis of daytime sleepiness in physicians after night duties. International Journal of Psychophysiology. 2009; 72:307-13. PMid:19452604. http:// dx.doi.org/10.1016/j.ijpsycho.2009.01.008

Wong KKW, Wan WY, Kaye SB. Blinking and operating: cognition versus vision. British Journal of Ophthalmology. 2001. 


\section{Autores}

\section{John Kennedy Schettino de Souza}

Programa de Pós-graduação em Engenharia Elétrica, Universidade Federal de Minas Gerais - UFMG, Av. Antônio Carlos, 6627, CEP 31270-010, Belo Horizonte, MG, Brasil.

\section{Giuliano Emerenciano Ginani, Sabine Pompéia}

Departamento de Psicobiologia, Universidade Federal de São Paulo - UNIFESP, Rua Napoleão de Barros, 1038, Vila Clementino, CEP 04024-003, São Paulo, SP, Brasil.

\section{Jerome Baron}

Programa de Pós-graduação em Engenharia Elétrica, Universidade Federal de Minas Gerais - UFMG, Av. Antônio Carlos, 6627, CEP 31270-010, Belo Horizonte, MG, Brasil

Departamento de Fisiologia e Biofísica, Instituto de Ciências Biológicas, Universidade Federal de Minas Gerais - UFMG, Av. Antônio Carlos, 6627, CEP 31270-010, Belo Horizonte, MG, Brasil.

\section{Carlos Julio Tierra-Criollo*}

Programa de Pós-graduação em Engenharia Elétrica, Universidade Federal de Minas Gerais - UFMG, Av. Antônio Carlos, 6627, CEP 31270-010, Belo Horizonte, MG, Brasil

Programa de Pós-graduação em Engenharia Biomédica - COPPE, Universidade Federal de Rio de Janeiro - UFRJ, Av. Horácio Macedo, 2030, CEP 21941-914, Rio de Janeiro, RJ, Brasil. 